\title{
On Waring's problem in finite fields
}

\author{
by \\ Arne Winterhof (Braunschweig)
}

1. Introduction. Let $g\left(k, p^{n}\right)$ be the smallest $s$ such that every element of $\mathbb{F}_{p^{n}}$ is a sum of $s k$ th powers in $\mathbb{F}_{p^{n}}$.

In Section 2 we summarize the basic results on $g\left(k, p^{n}\right)$. In Section 3 we generalize Dodson's upper bound for small $k$ ([5], Lemma 2.5.4):

$$
g(k, p)<\lfloor 8 \ln p\rfloor+1 ; \quad k \mid p-1, p / 2<k^{2}<p,
$$

and deduce

$$
g\left(k, p^{n}\right) \leq\lfloor 32 \ln k\rfloor+1 \quad \text { for } p^{n}>k^{2} .
$$

The object of Section 4 is to investigate to what extent Waring's problem for $\mathbb{F}_{p^{n}}$ can be reduced to the problem for $\mathbb{F}_{p}$. It is proven that if $g\left(k, p^{n}\right)$ exists, then

$$
g\left(k, p^{n}\right) \leq n g(d, p) ; \quad d=\frac{k}{\left(k,\left(p^{n}-1\right) /(p-1)\right)}, k \mid p^{n}-1 .
$$

It is well known (see [3]) that

$$
g(k, p) \leq\lfloor k / 2\rfloor+1 ; \quad k<(p-1) / 2 .
$$

[15], Theorem 1, implies that if $g\left(k, p^{n}\right)$ exists and $p$ is odd, then $g\left(k, p^{n}\right) \leq$ $\lfloor k / 2\rfloor+1$ for $k<\left(p^{n}-1\right) / 2$. Whether $p$ has to be odd has not been known yet. In Section 5 we show that $p$ need not be odd.

2. Basic results on $g\left(k, p^{n}\right)$. Every $\left(k, p^{n}-1\right)$ th power is at the same time a $k$ th power. Hence,

$$
g\left(k, p^{n}\right)=g\left(\left(k, p^{n}-1\right), p^{n}\right) .
$$

It is sufficient to restrict ourselves to the case

$$
k \mid p^{n}-1 .
$$

Remember that the multiplicative group $\mathbb{F}_{p^{n}}^{*}$ is cyclic. Hence

$$
g\left(k, p^{n}\right)=1 \Leftrightarrow k=1 .
$$

1991 Mathematics Subject Classification: 11P05, 11 T99. 
Since $L:=\left\{x_{1}^{k}+\ldots+x_{s}^{k} \mid x_{1}, \ldots, x_{s} \in \mathbb{F}_{p^{n}}, s \in \mathbb{N}\right\}$ is a field ([16], Lemma 1), $g\left(k, p^{n}\right)$ exists if and only if $L$ is not a proper subfield of $\mathbb{F}_{p^{n}}$, and thus

$$
g\left(k, p^{n}\right) \text { exists if and only if } \frac{p^{n}-1}{p^{d}-1} \nmid k \text { for all } n \neq d \mid n .
$$

This result is essentially that of [1], Theorem G.

We shall suppose that from now on $g\left(k, p^{n}\right)$ exists.

Let $A_{i}=\left\{z_{1}^{k}+\ldots+z_{i}^{k} \mid z_{1}, \ldots, z_{i} \in \mathbb{F}_{p^{n}}\right\}$. If $A_{i} \varsubsetneqq A_{i+1}$ then $y \in A_{i+1} \backslash A_{i}$ implies $x y \in A_{i+1} \backslash A_{i}$ for each $0 \neq x \in A_{1}$, so that

$$
\left|A_{i+1}\right| \geq\left|A_{i}\right|+\left|A_{1}\right|-1=\left|A_{i}\right|+\frac{p^{n}-1}{k} .
$$

Hence in the chain $A_{1} \subset A_{2} \subset \ldots \subset A_{s}=\mathbb{F}_{p^{n}}$ there are at most $k-1$ strict inclusions and therefore

$$
g\left(k, p^{n}\right) \leq k,
$$

which is a specialization of [10], Théorème 7.14.

Equality holds for the following examples:

$$
g\left(1, p^{n}\right)=1, \quad g\left(2, p^{n}\right)=2, \quad g\left(\frac{p-1}{2}, p\right)=\frac{p-1}{2}, \quad g(p-1, p)=p-1 .
$$

Since $\left|A_{s}\right| \leq\left(\frac{p^{n}-1}{k}+1\right)^{s}$, we get a trivial lower bound for $g\left(k, p^{n}\right)$ :

$$
g\left(k, p^{n}\right) \geq\left\lceil\frac{\ln p^{n}}{\ln \left(\frac{p^{n}-1}{k}+1\right)}\right\rceil .
$$

For $n=1$ the following results are well known:

(8) $\quad g(k, p) \leq 68(\ln k)^{2} k^{1 / 2} ; \quad p>2 k+1 \quad[7]$,

(9) $\quad g(k, p) \leq\lfloor k / 2\rfloor+1 ; \quad p>2 k+1 \quad[3]$,

(10) $g(k, p) \leq\left(1+\frac{2 k^{2}}{p-1}\right)\left(1+\left\lfloor 2 \log _{2} p\right\rfloor\right) ; \quad p>k^{3 / 2} \quad[2]$,

(11) $\quad g(k, p) \leq 170 \frac{k^{7 / 3}}{(p-1)^{4 / 3}} \ln p ; \quad p \leq k^{7 / 4}+1 \quad[8]$,

(12) $g(k, p) \leq c_{\varepsilon}(\ln k)^{2+\varepsilon} ; \quad k \geq 2, p \geq \frac{k \ln k}{(\ln (\ln k+1))^{1-\varepsilon}}, \varepsilon>0 \quad$ [11],

(13) $g(k, p) \leq c_{\varepsilon} ; \quad k<p^{2 / 3-\varepsilon}, \varepsilon>0 \quad[9]$.

3. Extension of Dodson's bound for small $k$. Now we consider the case $0<(k-1)^{2}<p^{n}$. In this case $g\left(k, p^{n}\right)$ exists. 
The number $N_{s}(b) ; b \in \mathbb{F}_{p^{n}}^{*}$, of solutions of the equation

$$
x_{1}^{k}+\ldots+x_{s}^{k}=b ; \quad x_{1}, \ldots, x_{s} \in \mathbb{F}_{p^{n}},
$$

can be expressed in terms of Jacobi sums ([12], Theorem 6.34)

$$
N_{s}(b)=p^{n(s-1)}+\sum_{j_{1}, \ldots, j_{s}=1}^{k-1} \lambda^{j_{1}+\ldots+j_{s}}(b) J\left(\lambda^{j_{1}}, \ldots, \lambda^{j_{s}}\right),
$$

where $\lambda$ is a multiplicative character of $\mathbb{F}_{p^{n}}$ of order $k$.

Using the fact that

$$
\left|J\left(\lambda^{j_{1}}, \ldots, \lambda^{j_{s}}\right)\right|= \begin{cases}p^{n(s-1) / 2} & \text { if } \lambda^{j_{1}+\ldots+j_{s}} \text { is non-trivial, } \\ p^{n(s-2) / 2} & \text { if } \lambda^{j_{1}+\ldots+j_{s}} \text { is trivial }\end{cases}
$$

([12], Theorem 5.22), we obtain

$$
\left|N_{s}(b)-p^{n(s-1)}\right| \leq(k-1)^{s} p^{n(s-1) / 2}
$$

and in particular

$$
N_{s}(b) \geq p^{n(s-1)}-(k-1)^{s} p^{n(s-1) / 2} .
$$

Hence,

$$
g\left(k, p^{n}\right) \leq s \quad \text { for } p^{n(s-1)}>(k-1)^{2 s} .
$$

For $s=2$ this is Small's [14] result.

If $0<\theta(k-1)^{2} \leq p^{n}$ for $\theta>1$, then

$$
s>\frac{\ln \theta(k-1)^{2}}{\ln \theta} \geq \frac{\ln p^{n}}{\ln \left(p^{n} /(k-1)^{2}\right)} \quad \text { implies } p^{n(s-1)}>(k-1)^{2 s},
$$

and thus

$$
g\left(k, p^{n}\right) \leq\left\lfloor\frac{\ln \theta(k-1)^{2}}{\ln \theta}\right\rfloor+1 \quad \text { for } 0<\theta(k-1)^{2} \leq p^{n} ; \theta>1 .
$$

We define

$$
S(b)=\sum_{x \in \mathbb{F}_{p^{n}}} \psi\left(b x^{k}\right),
$$

where $\psi(x)=e^{\frac{2 \pi i}{p} \operatorname{Tr}(x)}$ denotes the additive canonical character. We denote by $\sum_{b}^{*}$ a summation in which $b \neq 0$ runs through a set of representatives, one from each of the $k-1$ non-power classes and one from the $k$ th power class.

LEMMA 1.

$$
\sum_{b}^{*}|S(b)|^{2}=k(k-1) p^{n}
$$


Proof. The deduction is the same as for Dodson's Lemma 2.5.1. We have

$$
\sum_{b \in \mathbb{F}_{p^{n}}}|S(b)|^{2}=\sum_{x, y \in \mathbb{F}_{p^{n}}} \sum_{b \in \mathbb{F}_{p^{n}}} \psi\left(b\left(x^{k}-y^{k}\right)\right)=p^{n} M,
$$

where $M$ denotes the number of solutions of $x^{k}=y^{k}$ in $\mathbb{F}_{p^{n}}$. Since $M=$ $1+\left(p^{n}-1\right) k$ and $S(0)=p^{n}$ we obtain

$$
\sum_{b \in \mathbb{F}_{p^{n}}^{*}}|S(b)|^{2}=(k-1) p^{n}\left(p^{n}-1\right) .
$$

The lemma follows since $S(b)$ has the same value for each element of the same class.

Lemma 2. Suppose that $x_{1}^{k}+\ldots+x_{s}^{k}$ does not represent every element of $\mathbb{F}_{p^{n}}$. Then there exist some $c \in \mathbb{F}_{p^{n}}^{*}$ such that

$$
|S(m c)|>p^{n}\left(1-m^{2} \frac{\ln p^{n}}{s}\right) ; \quad m=1, \ldots, p-1 .
$$

Proof. The proof is a direct extension of Dodson's proof for Lemma 2.5.2. Verify that

$$
N_{s}(b)=p^{-n} \sum_{x_{1}, \ldots, x_{s} \in \mathbb{F}_{p^{n}}} \sum_{t \in \mathbb{F}_{p^{n}}} \psi\left(t\left(x_{1}^{k}+\ldots+x_{s}^{k}-b\right)\right)=p^{-n} \sum_{t \in \mathbb{F}_{p^{n}}} S(t)^{s} \psi(-t b)
$$

and suppose that there exists a $b \in \mathbb{F}_{p^{n}}$ such that $N_{s}(b)=0$. Hence we get

$$
\sum_{t \in \mathbb{F}_{p^{n}}^{*}} S(t)^{s} \psi(-t b)=-p^{n s} .
$$

It follows that there exists an element $c \in \mathbb{F}_{p^{n}}^{*}$ such that

$$
|S(c)|^{s} \geq \frac{p^{n s}}{p^{n}-1}>p^{n(s-1)},
$$

whence

$$
|S(c)|>p^{n} \exp \left(-\frac{\ln p^{n}}{s}\right)>p^{n}\left(1-\frac{\ln p^{n}}{s}\right),
$$

which is the result for $m=1$.

For some real $\vartheta$ we have

$$
|S(c)|=\sum_{x \in \mathbb{F}_{p^{n}}} \exp \left(\frac{2 \pi i}{p}\left(\operatorname{Tr}\left(c x^{k}\right)-\vartheta\right)\right)
$$

and thus

$$
\sum_{x \in \mathbb{F}_{p^{n}}} \cos \left(\frac{2 \pi}{p}\left(\operatorname{Tr}\left(c x^{k}\right)-\vartheta\right)\right)>p^{n}\left(1-\frac{\ln p^{n}}{s}\right)
$$


whence

$$
\sum_{x \in \mathbb{F}_{p^{n}}} \sin ^{2}\left(\frac{\pi}{p}\left(\operatorname{Tr}\left(c x^{k}\right)-\vartheta\right)\right)<\frac{p^{n} \ln p^{n}}{2 s} .
$$

Since $|\sin m \varphi| \leq|m \sin \varphi|$ and $\operatorname{Tr}(m x)=m \operatorname{Tr}(x)$ for $m=1, \ldots, p-1$, we deduce that

$$
\sum_{x \in \mathbb{F}_{p^{n}}} \sin ^{2}\left(\frac{\pi}{p}\left(\operatorname{Tr}\left(m c x^{k}\right)-m \vartheta\right)\right)<\frac{m^{2} p^{n} \ln p^{n}}{2 s},
$$

whence

$$
\sum_{x \in \mathbb{F}_{p^{n}}} \cos \left(2 \frac{\pi}{p}\left(\operatorname{Tr}\left(m c x^{k}\right)-m \vartheta\right)\right)>p^{n}\left(1-\frac{m^{2} \ln p^{n}}{s}\right),
$$

and thus

$$
|S(m c)|>p^{n}\left(1-\frac{m^{2} \ln p^{n}}{s}\right) .
$$

Lemma 3. Suppose that 2 is a $k$ th power in $\mathbb{F}_{p^{n}}$ and $g\left(k, p^{n}\right)$ exists. Then

$$
g\left(k, p^{n}\right)<n\left(\left\lfloor\frac{\ln p}{\ln 2}\right\rfloor+1\right) .
$$

Proof. If $g\left(k, p^{n}\right)$ exists, then there exists a basis $\left\{b_{1}, \ldots, b_{n}\right\}$ of $k$ th powers. Let $x=a_{1} b_{1}+\ldots+a_{n} b_{n}$ be any element of $\mathbb{F}_{p^{n}} ; 0 \leq a_{i}<p$, $i=1, \ldots, n$. For $i=1, \ldots, n$ we can express $a_{i}$ as

$a_{i}=a_{i, 0}+a_{i, 1} 2+\ldots+a_{i, h_{i}} 2^{h_{i}} ; \quad a_{i, j} \in\{0,1\}, j=0, \ldots, h_{i}-1, a_{i, h_{i}}=1$. Since $2^{h_{i}} \leq a_{i}<p, x$ is a sum of at most $\left(h_{1}+1\right)+\ldots+\left(h_{n}+1\right)<n\left(\left\lfloor\frac{\ln p}{\ln 2}\right\rfloor+1\right)$ $k$ th powers.

Lemma 4. If $p^{n}>k^{2}$, then $g\left(k, p^{n}\right)<\left\lfloor 8 \ln p^{n}\right\rfloor+1$.

Proof. We suppose that for $s=\left\lfloor 8 \ln p^{n}\right\rfloor+1$ there exists an element $b \in \mathbb{F}_{p^{n}}$ that is not of the form $b=x_{1}^{k}+\ldots+x_{s}^{k}$ and obtain a contradiction.

By Lemma 2 there exists $c \in \mathbb{F}_{p^{n}}^{*}$ such that

$$
|S(c)|>p^{n}\left(1-\frac{\ln p^{n}}{s}\right)>\frac{7}{8} p^{n} \quad \text { and } \quad|S(2 c)|>p^{n}\left(1-\frac{4 \ln p^{n}}{s}\right)>\frac{1}{2} p^{n} .
$$

If 2 is not a $k$ th power then $c$ and $2 c$ are representatives of two different classes in the sum of Lemma 1 . Since $k^{2}<p^{n}$ this gives

$$
p^{2 n}<\left(\frac{7}{8}\right)^{2} p^{2 n}+\left(\frac{1}{2}\right)^{2} p^{2 n} \leq k(k-1) p^{n}<p^{2 n} .
$$

Hence 2 must be a $k$ th power and Lemma 3 implies that $b$ is a sum of $n(\lfloor\lfloor\ln p\rfloor+1) \leq s k$ th powers.

Corollary 1. If $p^{n} / \theta \leq k^{2}<p^{n}$ for some $\theta>1$, then

$$
g\left(k, p^{n}\right) \leq\left\lfloor 8 \ln \theta k^{2}\right\rfloor+1 .
$$


From Corollary 1 with $\theta=k^{2}$ and (14) with $s=2$ we get:

TheOREM 1. $g\left(k, p^{n}\right) \leq\lfloor 32 \ln k\rfloor+1$ for $p^{n}>k^{2}$.

This generalizes [6], p. 151, (6).

\section{A relation between $g\left(k, p^{n}\right)$ and $g(d, p)$}

TheOREM 2. If $g\left(k, p^{n}\right)$ exists, then

$$
g\left(k, p^{n}\right) \leq n g(d, p) ; \quad d=\frac{k}{\left(k, \frac{p^{n}-1}{p-1}\right)}=\frac{p-1}{\left(\frac{p^{n}-1}{k}, p-1\right)} .
$$

Proof. If $g\left(k, p^{n}\right)$ exists, then there exists a basis $\left\{b_{1}, \ldots, b_{n}\right\}$ of $\mathbb{F}_{p^{n}}$ over $\mathbb{F}_{p}$ consisting of $k$ th powers.

The $k$ th powers are exactly the $\frac{p^{n}-1}{k}$ th roots of unity. Thus, the $k$ th powers of elements of $\mathbb{F}_{p^{n}}^{*}$ in $\mathbb{F}_{p}^{*}$ are exactly the $\left(\frac{p^{n}-1}{k}, p-1\right)$ th roots of unity which are the $d$ th powers of elements of $\mathbb{F}_{p}^{*}$. Hence, all elements of $\mathbb{F}_{p}$ are sums of $g(d, p) k$ th powers of elements of $\mathbb{F}_{p^{n}}$, so that all elements of the form $b_{i} a ; a \in \mathbb{F}_{p}, i=1, \ldots, n$, are sums of $g(d, p) k$ th powers. Thus an arbitrary element $x=a_{1} b_{1}+\ldots+a_{n} b_{n} \in \mathbb{F}_{p^{n}} ; a_{i} \in \mathbb{F}_{p}, i=1, \ldots, n$, is a sum of $n g(d, p) k$ th powers.

\section{Extension of the Chowla/Mann/Straus bound}

Theorem 3. If $g\left(k, 2^{n}\right)$ exists, then $g\left(k, 2^{n}\right) \leq(k+1) / 2$. for

Proof. By Theorem 2 we have $g\left(k, 2^{n}\right) \leq n$, which implies the result

$$
n \leq(k+1) / 2 .
$$

Moreover, (14) with $s=2$ implies the result for

$$
2^{n}>(k-1)^{4} \text {. }
$$

Hence it is sufficient to consider $2 \leq n \leq 21$. By (4), (16) and (17) we have 12 pairs $\left(k, 2^{n}\right)$ to investigate: $g\left(3,2^{4}\right), g\left(7,2^{6}\right), g\left(5,2^{8}\right), g\left(7,2^{9}\right)$, $g\left(11,2^{10}\right), g\left(9,2^{12}\right), g\left(13,2^{12}\right), g\left(15,2^{12}\right), g\left(21,2^{12}\right), g\left(17,2^{16}\right), g\left(27,2^{18}\right)$, and $g\left(33,2^{20}\right)$.

For $k \geq 5$ and $2^{n}>(k-1)^{3}$ or $k \geq 7$ and $2^{3 n}>(k-1)^{8}$ we get the result by (14). Hence only $g\left(3,2^{4}\right)$ and $g\left(7,2^{6}\right)$ are undecided. It is well known that for $p^{n} \neq 4$ and 7 every element of $\mathbb{F}_{p^{n}}$ is a sum of two cubes (see [13]), which implies $g\left(3,2^{4}\right)=2$. As in the proof of Theorem 2 we get $g\left(7,2^{6}\right) \leq 3 g\left(1,2^{2}\right)$, which completes the proof.

REMARK. For small $k$ it is shown in [4] that $g\left(k, p^{n}\right) \leq\lfloor k / 2\rfloor+1$ for $k<\min \left(p,\left(p^{n}-1\right) / 2\right)$.

For arbitrary $k$ but $p \neq 2$, [15], Theorem 1 , implies $g\left(k, p^{n}\right) \leq\lfloor k / 2\rfloor+1$ for $k<\left(p^{n}-1\right) / 2$. 


\section{References}

[1] M. Bhaskaran, Sums of mth powers in algebraic and abelian number fields, Arch. Math. (Basel) 17 (1966), 497-504; Correction, ibid. 22 (1971), 370-371.

[2] J. D. Bovey, A new upper bound for Waring's problem mod p, Acta Arith. 32 (1977), 157-162.

[3] S. Chowla, H. B. Mann and E. G. Straus, Some applications of the CauchyDavenport theorem, Norske Vid. Selsk. Forh. Trondheim 32 (1959), 74-80.

[4] G. T. Diderrich and H. B. Mann, Representations by $k$-th powers in $G F(q)$, J. Number Theory 4 (1972), 269-273.

[5] M. M. Dodson, Homogeneous additive congruences, Philos. Trans. Roy. Soc. London Ser. A 261 (1967), 163-210.

6] - On Waring's problem in GF[p], Acta Arith. 19 (1971), 147-173.

[7] M. M. Dodson and A. Tietäväinen, A note on Waring's problem in GF $[p]$, ibid. 30 (1976), 159-167.

[8] A. Garcia and J. F. Voloch, Fermat curves over finite fields, J. Number Theory 30 (1988), 345-356.

[9] D. R. Heath-Brown and S. Konyagin, New bounds for Gauss sums derived from kth powers and for Heilbronn's exponential sum, submitted to Quart. J. Math. Oxford.

[10] J. R. Joly, Sommes de puissances d-ièmes dans un anneau commutatif, Acta Arith. 17 (1970), 37-114.

[11] S. V. Konyagin, On estimates of Gaussian sums and Waring's problem for a prime modulus, Trudy Mat. Inst. Steklov. 198 (1992), 111-124 (in Russian); English transl.: Proc. Steklov Inst. Math. 1994, no. 1, 105-117.

[12] R. Lidl and H. Niederreiter, Finite Fields, Encyclopedia Math. Appl. 20, Addison-Wesley, 1983.

[13] S. Singh, Analysis of each integer as sum of two cubes in a finite integral domain, Indian J. Pure Appl. Math. 6 (1975), 29-35.

[14] C. Small, Sums of powers in large finite fields, Proc. Amer. Math. Soc. 65 (1977), $35-36$.

[15] A. Tietäväinen, On diagonal forms over finite fields, Ann. Univ. Turku Ser. A I 118 (1968), $10 \mathrm{pp}$.

[16] L. Tornheim, Sums of $n$-th powers in fields of prime characteristic, Duke Math. J. 4 (1938), 359-362.

Institut für Algebra und Zahlentheorie

TU Braunschweig

Pockelsstr. 14

38106 Braunschweig, Germany

E-mail: A.Winterhof@tu-bs.de 